\title{
A method for including external feed in depletion calculations with CRAM and implementation into ORIGEN
}

\author{
A. E. Isotalo ${ }^{\mathrm{a}, \mathrm{b}, *}$, W. A. Wieselquist ${ }^{\mathrm{a}}$ \\ ${ }^{a}$ Oak Ridge National Laboratory, P.O. Box 2008, Oak Ridge, TN 37831-6172, USA \\ ${ }^{b}$ Aalto University, Department of Applied Physics, P.O.Box 14100, FI-00076 AALTO, \\ Finland
}

\begin{abstract}
A method for including external feed with polynomial time dependence in depletion calculations with the Chebyshev Rational Approximation Method (CRAM) is presented and the implementation of CRAM to the ORIGEN module of the SCALE suite is described. In addition to being able to handle time-dependent feed rates, the new solver also adds the capability to perform adjoint calculations. Results obtained with the new CRAM solver and the original depletion solver of ORIGEN are compared to high precision reference calculations, which shows the new solver to be orders of magnitude more accurate. Furthermore, in most cases, the new solver is up to several times faster due to not requiring similar substepping as the original one.
\end{abstract}

Keywords: CRAM, ORIGEN, depletion, external feed, source term

\footnotetext{
*Corresponding author

Email address: aarno.isotalo@aalto.fi (A. E. Isotalo)
} 


\section{Introduction}

Calculating changes in the isotopic composition of nuclear fuel or other materials under irradiation or during decay is essential for a wide range of design, operational, and licensing analyses in applications ranging from reactor physics to waste management and safeguards. These applications require methods and codes that enable changes in compositions to be accurately and reliably calculated for widely different initial compositions, neutron spectra, flux levels, and time scales from seconds after fission to tens of thousands of years after fuel discharge. The number of nuclides to be accounted for also varies by application, but often it is either necessary or desirable to consider a large systems consisting of a thousand or more nuclides with widely varying decay constants and up to tens of thousands of possible transitions between them. Although external feed (i.e., the continuous introduction of material into the observed system from outside) is not relevant in the most typical applications of decay and depletion calculations, modeling it is necessary to easily and robustly handle certain systems. Examples of such systems in-

This manuscript has been authored by UT-Battelle, LLC under Contract No. DEAC05-00OR22725 with the U.S. Department of Energy. The United States Government retains and the publisher, by accepting the article for publication, acknowledges that the United States Government retains a non-exclusive, paid-up, irrevocable, world-wide license to publish or reproduce the published form of this manuscript, or allow others to do so, for United States Government purposes. The Department of Energy will provide public access to these results of federally sponsored research in accordance with the DOE Public Access Plan(http://energy.gov/downloads/doe-public-access-plan). 
clude liquid fueled reactors and storage and reprocessing facilities, where the in-flow of material affects reactivity, decay heat, and activity loads.

In the SCALE nuclear systems modeling and simulation suite (Oak Ridge National Laboratory, 2011), all decay and depletion calculations are handled by ORIGEN (Gauld et al., 2011). The original depletion solver of ORIGEN uses a secular equilibrium approximation and the Bateman solution of linear chains for handling shortlived nuclides, and a power series approximation of the matrix exponential of a reduced coefficient matrix for long-lived nuclides. While the linear chains and matrix exponential can be solved accurately when independent, accounting for the effects of long-lived nuclides on the short-lived ones and vice versa requires additional approximations and leads to a loss of accuracy for some nuclides in the hybrid method. This method has usually been referred to as the matrix exponential method or the ORIGEN method. However, as a part of the SCALE modernization campaign, the ORIGEN code now has more than one solver. To make a distinction between these solvers, the original method of solution is referred to as MATREX.

While the MATREX solver has continued to perform adequately, there has also been significant development in depletion algorithms. CRAM (Pusa and Leppänen, 2010) is perhaps the most promising of the new methods. It has been shown to yield very accurate results while still being among the fastest methods for solving the decay and depletion of large systems of nuclides (e.g., Isotalo and Aarnio, 2011; Pusa, 2013b). However, CRAM cannot model external feed. Typical depletion calculations of solid reactor fuels do not require such capability, so this has not been an issue. However, ORIGEN and its solvers must be able to provide all the depletion capabilities of SCALE, which 
is also used for applications with external feed.

In this paper we present a method for including a source term in depletion calculations with CRAM. The methodology allows accurate modeling of external feed with polynomial or exponentially decaying time dependence, although the usable polynomial order is limited by the order of the CRAM approximation. Results obtained with the new CRAM solver of ORIGEN with and without external feed are also presented and compared with the MATREX solver when applicable.

\section{Theory}

The equations governing the decay and transmutation of an arbitrary mixture of $n$ different nuclides in a homogenized material region can be written in a matrix form as

$$
\frac{d \boldsymbol{x}}{d t}=A \boldsymbol{x}+\boldsymbol{s}
$$

where $\boldsymbol{x}=\left[\begin{array}{lll}x_{1} & \cdots & x_{n}\end{array}\right]^{\top}, \boldsymbol{s}=\left[\begin{array}{lll}s_{1} & \cdots & s_{n}\end{array}\right]^{\top}$, and $x_{i}$ and $s_{i}$ are the atomic density and source term of nuclide $i$. A positive source term represents an external feed of nuclides into the system, while a negative one would represent the removal of a fixed number of nuclei per second. The $n$-by- $n$ matrix $A$ is called the transition or coefficient matrix. The diagonal elements of the matrix are the removal rates of each nuclide while the off-diagonal elements are the production rates from other nuclides:

$$
a_{i, i}=-\lambda_{i}-\phi \sum_{r} \sigma_{i, r}
$$

and

$$
a_{i, j \neq i}=b_{i, j} \lambda_{i}+\sum_{r} y_{i, j, r} \sigma_{i, r} \phi
$$


where $\lambda_{i}$ is the decay constant of nuclide $i, b_{i, j}$ the branching ratio from nuclide $j$ to nuclide $i, \phi$ the one-group neutron flux, $\sigma_{i, r}$ the microscopic onegroup cross section of reaction $r$ of nuclide $i$, and $y_{i, j, r}$ the yield of nuclide $i$ when nuclide $j$ undergoes reaction $r$. In general, $\phi, \sigma_{i, r}, y_{i, j, r}$, and $s$ depend on time, but they are approximated constant over each step of the calculation when the equations are solved (e.g., Isotalo, 2013).

\section{1. $C R A M$}

If $\boldsymbol{s}=0$, then Eq. (1) has a formal solution $\boldsymbol{x}(t)=e^{A t} \boldsymbol{x}(0)$, which employs the matrix exponential notation. So-called matrix exponential methods are based on different numerical approximations for evaluating the matrix exponential $e^{A t}$. CRAM (Pusa and Leppänen, 2010) is a matrix exponential method based on the observation that the eigenvalues of the coefficient matrix are clustered around the negative real axis. This is exploited by making a Chebyshev rational approximation of the exponential function for the interval $(-\infty, 0]$. The resulting rational function is then decomposed into a pole-residue form. When the denominator and numerator orders of the rational approximation are selected equal and even, the poles form conjugate pairs, and the imaginary parts cancel out for a real valued variable. When applied to the matrix exponential, the approximation becomes

$$
\boldsymbol{x}(t) \approx a_{0} \boldsymbol{x}(0)+2 \operatorname{Re}\left[\sum_{i=1}^{k / 2} a_{i}\left(A t+\theta_{i} I\right)^{-1}\right] \boldsymbol{x}(0)
$$

where $a_{0}$ is the limiting value of the approximation at infinity, $a_{i}$ and $\theta_{i}$ are the residues and poles, and $k$ is the order of the denominator and numerator polynomials, i.e., the order of the CRAM method. Due to the sparse 
structure of $A$, the matrix inversions can be calculated efficiently by solving systems of the form $\left(A t+\theta_{i} I\right) \boldsymbol{y}=\boldsymbol{x}(0)$ for $\boldsymbol{y}$ via Gaussian elimination or LU-decomposition. The coefficients (poles and residues) do not depend on $A, t$ or $\boldsymbol{x}$ and can thus be precalculated and tabulated.

Due to numerical problems, the partial fraction decomposition used in Eq. (4) is unsuited for evaluating order $k>16$ approximations (Pusa, 2014). Higher order approximations may be evaluated using a different form (Pusa, 2014), but they are not considered in this work.

\subsection{Constant source term}

CRAM can only evaluate the matrix exponential solution of the homogeneous system without a source term and thus is not directly applicable to the more general inhomogeneous system in Eq. (1). With a constant source term it is, however, straightforward to turn the inhomogeneous system into a homogeneous one by representing the source term as production from a dummy nuclide that has no removal:

$$
A \boldsymbol{x}+\boldsymbol{s} \widehat{=} \tilde{A} \tilde{\boldsymbol{x}}=\left[\begin{array}{cccc}
a_{1,1} & \cdots & a_{1, n} & s_{1} \\
\vdots & \ddots & \vdots & \vdots \\
a_{n, 1} & \cdots & a_{n, n} & s_{n} \\
0 & 0 & 0 & 0
\end{array}\right]\left[\begin{array}{c}
x_{1} \\
\vdots \\
x_{n} \\
d_{0}
\end{array}\right]
$$

with the initial condition

$$
\tilde{\boldsymbol{x}}(0)=\left[\begin{array}{llll}
x_{1}(0) & \cdots & x_{n}(0) & 1
\end{array}\right]^{\top} .
$$

The solution to this homogenized system is $\tilde{\boldsymbol{x}}(t)=e^{\tilde{A} t} \tilde{\boldsymbol{x}}(0)$, which can be evaluated with CRAM. Because the entire last row is zero, $d_{0}(t)=1$ over the 
entire step, and the last column of $\tilde{A}$ gives nuclide $i$ a constant source term (i.e. feed rate) of $s_{i}$.

\subsection{Non-constant source term}

Expanding on the above approach, it is possible to introduce a timedependent source term by building it up from dummy nuclides whose concentrations are made to change in a specific manner. For an order $m$ polynomial source term, $m+1$ dummy nuclides with concentrations $1, t, \ldots, t^{m}$ are required. This can be achieved by amending the system as shown below:

$$
A \boldsymbol{x}+\boldsymbol{s} \widehat{=} \tilde{A} \tilde{\boldsymbol{x}}=\left[\begin{array}{cccccccc}
a_{1,1} & \cdots & a_{1, n} & s_{1,0} & s_{1,1} & s_{1,2} & \cdots & s_{1, m} \\
\vdots & \ddots & \vdots & \vdots & \vdots & \vdots & \ddots & \vdots \\
a_{n, 1} & \cdots & a_{n, n} & s_{n, 0} & s_{n, 1} & s_{n, 2} & \cdots & s_{n, m} \\
0 & \cdots & 0 & 0 & 0 & 0 & \cdots & 0 \\
0 & \cdots & 0 & 1 & 0 & 0 & \cdots & 0 \\
0 & \cdots & 0 & 0 & 2 & 0 & \cdots & 0 \\
\vdots & \ddots & \ddots & \ddots & \ddots & \ddots & \ddots & \vdots \\
0 & \cdots & 0 & 0 & 0 & 0 & m & 0
\end{array}\right]\left[\begin{array}{c}
x_{1} \\
\vdots \\
x_{n} \\
d_{0} \\
d_{1} \\
d_{2} \\
\vdots \\
d_{m}
\end{array}\right]
$$

with the initial condition

$$
\tilde{\boldsymbol{x}}(0)=\left[\begin{array}{lllllll}
x_{1}(0) & \cdots & x_{n}(0) & 1 & 0 & \cdots & 0
\end{array}\right]^{\top} .
$$

The last $m+1$ rows can be solved for $d_{i}=t^{i}$, which gives the external production rate of nuclide $i$ as

$$
s_{i}(t)=\sum_{j=0}^{m} s_{i, j} t^{j} .
$$


An exponential source term of the shape

$$
s_{i}(t)=\sum_{j=1} s_{i, j} e^{-c_{j} t}, \quad c_{j} \geq 0
$$

is possible by modifying the system to:

$$
A \boldsymbol{x}+\boldsymbol{s} \widehat{=} \tilde{A} \tilde{\boldsymbol{x}}=\left[\begin{array}{ccccccc}
a_{1,1} & \cdots & a_{1, n} & s_{1,1} & s_{1,2} & \cdots & s_{1, m} \\
\vdots & \ddots & \vdots & \vdots & \vdots & \ddots & \vdots \\
a_{n, 1} & \cdots & a_{n, n} & s_{n, 1} & s_{n, 2} & \cdots & s_{n, m} \\
0 & \cdots & 0 & -c_{1} & 0 & \cdots & 0 \\
\vdots & \ddots & \ddots & \ddots & \ddots & \ddots & \vdots \\
0 & \cdots & 0 & 0 & 0 & -c_{m-1} & 0 \\
0 & \cdots & 0 & 0 & 0 & 0 & -c_{m}
\end{array}\right]\left[\begin{array}{c}
x_{1} \\
\vdots \\
x_{n} \\
d_{1} \\
\vdots \\
d_{m-1} \\
d_{m}
\end{array}\right]
$$

with the initial condition

$$
\tilde{\boldsymbol{x}}(0)=\left[\begin{array}{llllll}
x_{1}(0) & \cdots & x_{n}(0) & 1 & \cdots & 1
\end{array}\right]^{\top} .
$$

A source term can mix polynomial and exponential terms simply by appending both of the above modifications to the coefficient matrix.

\subsection{Accuracy of CRAM for the homogenized system}

If $f(x)$ is an analytic scalar function on an open set containing the eigenvalues of the matrix $A t$ and $A=T J T^{-1}$ is the Jordan decomposition of $A$, then the corresponding matrix function $f(A t)$ can be defined as (Higham, 2008)

$$
f(A t)=T \operatorname{diag}\left[f\left(J_{\beta_{1}}\left(\lambda_{1} t\right)\right) \quad \cdots \quad f\left(J_{\beta_{b}}\left(\lambda_{b} t\right)\right)\right] T^{-1}
$$

where

$$
f\left(J_{\beta_{j}}\left(\lambda_{j} t\right)\right)=\sum_{\nu=0}^{\beta_{j}-1} \frac{f^{(\nu)}\left(\lambda_{j} t\right)}{\nu !} S_{\beta_{j}}^{\nu}
$$


$b$ is the number of Jordan blocks of $A, \beta_{j}$ are the sizes of the Jordan blocks and $\lambda_{j}$ the corresponding eigenvalues, $f^{(\nu)}$ is the $\nu$ :th derivative of $f$, and $S_{\beta_{j}}$ a $\beta_{j}$-by- $\beta_{j}$ matrix with ones on the first superdiagonal and zeroes everywhere else. While the difficulty of finding the Jordan decomposition makes this form impractical in actual calculations, it is equivalent with other definitions of matrix functions and can provide great insight.

When we approximate $f(A t)=e^{A t}$ with $r(A t)$, the resulting errors are $r(A t)-f(A t)=T \operatorname{diag}\left[r\left(J_{\beta_{1}}\left(\lambda_{1} t\right)\right)-f\left(J_{\beta_{1}}\left(\lambda_{1} t\right)\right) \quad \cdots \quad r\left(J_{\beta_{b}}\left(\lambda_{b} t\right)\right)-f\left(J_{\beta_{b}}\left(\lambda_{b} t\right)\right)\right] T^{-1}$

where we can simplify

$$
r\left(J_{\beta_{j}}\left(\lambda_{j} t\right)\right)-f\left(J_{\beta_{j}}\left(\lambda_{j} t\right)\right)=\sum_{\nu=0}^{\beta_{j}-1} \frac{r^{(\nu)}\left(\lambda_{j} t\right)-f^{(\nu)}\left(\lambda_{j} t\right)}{\nu !} S_{\beta_{j}}^{\nu} .
$$

In CRAM $f(A t)=e^{A t}$ is approximated with the Chebyshev rational approximation $r(A t)=\hat{r}_{k, k}(A t)$. The errors thus depend on how well $\hat{r}_{k, k}$ can approximate the first $\beta_{j}$ derivatives of the exponential function at $\lambda_{j} t$ for $j=1 \ldots b$. Physical $A$ only has Jordan blocks of size 1 and thus no dependency on the derivatives of $\hat{r}_{k, k}$, although the limited precision of library data means that this is not guaranteed to hold in practical applications (Pusa, 2013a). An exponential source term introduces distinct eigenvalues $-c_{j}$, so accuracy with it also depends on $\hat{r}_{k, k}\left(-c_{i} t\right)$. As long as $-c_{j} \leq 0$, overall accuracy is not significantly affected.

A polynomial source term is more complicated. Consider $\tilde{A}$ for a polynomial source and denote by $D$ its lower right $m+1$-by- $m+1$ block. Clearly the Jordan decomposition of $D$ is $T_{D} J_{D} T_{D}^{-1}$, where $T_{D}$ is skew diagonal with $[m !(m-1) ! \cdots \quad 0 !]$ and $J_{D}=S_{m+1}$. Because the diagonal block $D$ has a 
Jordan block of size $m+1$ for zero, $\tilde{A}$ must also have one of at least size $m+1$. On the other hand, zero is a semisimple eigenvalue of $A$ (Pusa and Leppänen, 2010), and the eigenpairs of $A$ with the vectors zero-padded are eigenpairs of $\tilde{A}$. This means that the size of any Jordan block of $\tilde{A}$ larger than 1 for the eigenvalue zero can be at most the number of zero eigenvalues outside $A$ (i.e., $m+1$ ). Thus the largest Jordan block of $\tilde{A}$ for zero must be exactly size $m+1$ and the rest size 1 .

With a constant source term (i.e. $m=0$ ) CRAM should be as accurate as without a source term, but the derivatives of $\hat{r}_{k, k}$ are progressively worse approximations of the derivatives of the exponential function, and accuracy deteriorates as the source term order is increased. Table 1 lists the relative error in the derivatives of order 6-16 approximations at zero. Relative errors roughly comparable in magnitude to the $m^{\text {th }}$ derivative are expected with order $m$ source term. As a rough rule of thumb, about one digit of accuracy is lost per source order. Since CRAM is extremely accurate, several digits can be lost while still being well below the uncertainties in nuclear data. Whether the source term is polynomial or exponential, the actual values of the coefficients $s_{i, j}$ do not affect the overall accuracy of the approximation, except through the highest order non-zero coefficient determining the order of a polynomial source.

Note that the source term handling and the above discussion on its accuracy do not rely on the pole-residue form in Eq. (4). Hence they should be equally applicable if an alternate functional form is used for CRAM (Pusa, 2014). The Jordan-block deduction also holds for any other functional approximation $g(A t)$ including, for example, all rational and polynomial ap- 
Table 1: Relative errors in the derivatives of the Chebyshev rational approximation at

\begin{tabular}{c|cccccc}
\hline & \multicolumn{5}{c}{ zero. } & \multicolumn{5}{c}{ Approximation order } \\
Derivative & 6 & 8 & 10 & 12 & 14 & 16 \\
\hline 0 & $-1.0 \times 10^{-6}$ & $-1.2 \times 10^{-8}$ & $-1.4 \times 10^{-10}$ & $-1.6 \times 10^{-12}$ & $-1.8 \times 10^{-14}$ & $-6.6 \times 10^{-15}$ \\
1 & $-5.3 \times 10^{-5}$ & $-8.0 \times 10^{-7}$ & $-1.1 \times 10^{-8}$ & $-1.6 \times 10^{-10}$ & $-2.1 \times 10^{-12}$ & $-2.8 \times 10^{-14}$ \\
2 & $-9.2 \times 10^{-4}$ & $-1.8 \times 10^{-5}$ & $-3.2 \times 10^{-7}$ & $-5.3 \times 10^{-9}$ & $-8.2 \times 10^{-11}$ & $-1.2 \times 10^{-12}$ \\
3 & $-9.7 \times 10^{-3}$ & $-2.5 \times 10^{-4}$ & $-5.4 \times 10^{-6}$ & $-1.1 \times 10^{-7}$ & $-1.9 \times 10^{-9}$ & $-3.3 \times 10^{-11}$ \\
4 & $-7.5 \times 10^{-2}$ & $-2.5 \times 10^{-3}$ & $-6.6 \times 10^{-5}$ & $-1.5 \times 10^{-6}$ & $-3.2 \times 10^{-8}$ & $-6.2 \times 10^{-10}$ \\
5 & $-4.6 \times 10^{-1}$ & $-2.0 \times 10^{-2}$ & $-6.3 \times 10^{-4}$ & $-1.7 \times 10^{-5}$ & $-4.2 \times 10^{-7}$ & $-9.2 \times 10^{-9}$ \\
6 & $-2.4 \times 10^{0}$ & $-1.3 \times 10^{-1}$ & $-5.0 \times 10^{-3}$ & $-1.6 \times 10^{-4}$ & $-4.5 \times 10^{-6}$ & $-1.1 \times 10^{-7}$ \\
7 & $-1.1 \times 10^{1}$ & $-7.3 \times 10^{-1}$ & $-3.4 \times 10^{-2}$ & $-1.3 \times 10^{-3}$ & $-4.1 \times 10^{-5}$ & $-1.2 \times 10^{-6}$ \\
8 & $-4.5 \times 10^{1}$ & $-3.7 \times 10^{0}$ & $-2.1 \times 10^{-1}$ & $-9.1 \times 10^{-3}$ & $-3.3 \times 10^{-4}$ & $-1.1 \times 10^{-5}$ \\
9 & $-1.7 \times 10^{2}$ & $-1.7 \times 10^{1}$ & $-1.1 \times 10^{0}$ & $-5.7 \times 10^{-2}$ & $-2.4 \times 10^{-3}$ & $-8.6 \times 10^{-5}$ \\
10 & $-5.4 \times 10^{2}$ & $-7.1 \times 10^{1}$ & $-5.6 \times 10^{0}$ & $-3.3 \times 10^{-1}$ & $-1.6 \times 10^{-2}$ & $-6.3 \times 10^{-4}$ \\
\hline
\end{tabular}


proximations. For such methods, the accuracy from a polynomial source term depends on the accuracies of $g^{(\nu)}(0)$ for $\nu=1 \ldots m$. This means that a constant source term should not affect the overall accuracy, and that the accuracy of any such method will deteriorate as the source order is increased.

\subsection{Adjoint calculation}

One of the motivations for implementing CRAM in ORIGEN was to solve the adjoint depletion equations (Williams, 1978):

$$
-\frac{d \boldsymbol{x}^{*}}{d \tau}=A^{\top} \boldsymbol{x}^{*}+\boldsymbol{s}^{*}(\tau), \quad \boldsymbol{x}^{*}\left(\tau_{f}\right)=\boldsymbol{x}_{f}^{*}
$$

which can be used for perturbation theory based sensitivity and depletion uncertainty analysis.

Adjoint calculations with CRAM are straightforward. By making a change of variable from $\tau$ to $t=\tau_{f}-\tau$, the adjoint problem can be brought to the form:

$$
\frac{d \boldsymbol{x}^{*}}{d t}=A^{\top} \boldsymbol{x}^{*}+\boldsymbol{s}^{*}(t), \quad \boldsymbol{x}^{*}(0)=\boldsymbol{x}_{f}^{*} .
$$

The only difference from the forward system is that the coefficient matrix is transposed. As transposing does not affect the eigenvalues or the Jordan blocks, the exponential of the transposed matrix is evaluated by CRAM as

accurately as that of the original one. Note that only $A$ is transposed. $\tilde{A}$ for the adjoint system thus contains $A^{\top}$ in the upper left block, $\boldsymbol{s}^{*}(t)$ on the right side, and the same lower left block as Eq. (7).

\subsection{Post-processing}

While the errors produced by CRAM are small in the absolute sense, relative errors can be unbounded for nuclides whose concentrations are dramatically reduced during a step (Pusa, 2013a,b). This situation is usually 
only encountered in decay calculations. Without a source term, an approximate lower limit for the magnitude of these errors is $\epsilon_{i} \approx H^{-k} x_{i}(0)$, where $k$ is the order of the CRAM approximation and $H \approx 9.3$ is the Halphen constant (Pusa, 2014). Thus if $x_{i}(t)$ is of the order of $H^{-k} x_{i}(0)$ or smaller, it contains no useful information. As these atomic densities are known to be wrong and may have unbounded relative errors, it may be desirable to remove them from the results by setting any $x_{i}(t)$ smaller than some limit to zero. Since the above error estimate is a lower limit, the post-processing limit should be higher than $H^{-k} x_{i}(0)$. In this work, a limit of $H^{-k+1} x_{i}(0)$ is used, but it is not guaranteed to be optimal in all applications.

When a source term is present, it is possible to calculate the contributions from it separately:

$$
e^{\tilde{A} t} \tilde{\boldsymbol{x}}(0)=e^{\tilde{A} t} \tilde{\boldsymbol{x}}_{\mathrm{ini}}(0)+e^{\tilde{A} t} \tilde{\boldsymbol{x}}_{\mathrm{src}}(0)
$$

where $\tilde{\boldsymbol{x}}_{\text {ini }}(0)=\left[\begin{array}{llll}\boldsymbol{x}(0)^{\top} & 0 & \cdots & 0\end{array}\right]^{\top}$ and $\tilde{\boldsymbol{x}}_{\text {src }}(0)=\hat{e}_{n+1}$, i.e., the $(n+1)$ st unit vector. The post-processing condition can then be applied to the first term. The computational cost of calculating the contributions separately depends on the method used for the matrix inversions in Eq. (4). If LUdecompositions are formed explicitly, as is done in this work, evaluating the components separately has only a modest effect on running time.

Note that this post-processing only considers errors that a nuclide contributes to itself. Thus it does not guarantee that the remaining results are accurate regardless of the limit chosen. In particular, it can never detect the errors for a nuclide whose initial concentration is zero. 


\section{Test Calculations}

A CRAM solver with a polynomial source term was implemented into ORIGEN using the sequential SuperLU library version 4.3 (Li, 2005) for solving the sparse linear systems arising from Eq. (4). In the implementation, the nuclides are sorted by ascending ZAI, and all pivoting and scaling are disabled in SuperLU. Order 2-16 CRAM coefficients were provided by Dr. Maria Pusa from the VTT Technical Research Centre of Finland. The post-processing to remove low concentration nuclides with large errors (Section 2.6) was also implemented but was not used in the test calculations except when otherwise noted.

\subsection{Reference Calculations}

Results obtained with CRAM and MATREX in tests without source term or with a constant source term were compared with reference calculations performed with a separate test program using the linear chains method with the general analytic solution (Cetnar, 2006), general analytic source term solution $^{1}$, passage-based chain termination (Isotalo and Aarnio, 2011) and the multiple precision floating point reliable (MPFR) library (Fousse et al., 2007). The only approximations in the test program are the chain termination and floating point arithmetics, both of which can be adjusted freely. The reference calculations used 500 bit mantissa (about 150 digits) and a cutoff

\footnotetext{
${ }^{1}$ An analytical solution for a chain with a constant source term for the first nuclide can be derived from the regular chain solution by representing the source term as the decay of an imaginary first nuclide and taking the limit as the decay constant of the imaginary nuclide approaches zero while its macroscopic decay rate remains constant.
} 
of $10^{-45}$, which give at least 14 correct digits at least 14 correct digits for all nuclides with atomic fractions above $10^{-30}$.

Reference solutions for the test cases that involve a time-dependent source term were calculated by evaluating $e^{\tilde{A} t} \tilde{\boldsymbol{x}}(0)$ with the variable precision arithmetics of MATLAB's symbolic toolbox. These calculations used 64 digit precision.

\subsection{Test cases}

All tests presented here used nuclear data from the w17_e40.arplib library distributed with SCALE 6.1. The library has precalculated burnup dependent one-group cross sections for a $17 \times 17$ Westinghouse PWR assembly with a 4 wt\% enrichment. It contains 1946 nuclides and 35013 reactions $^{2}$, although not all of the nuclides were produced in the tests.

Test calculations were performed with fresh and used fuel. Both compositions were used as the initial concentrations $x_{i}(0)$, as constant external feed rates of $s_{i}=x_{i}(0) / \Delta t$, where $\Delta t$ is the length of the depletion or decay step, or as both. The composition of the fresh fuel is $\mathrm{UO}_{2}$ with an enrichment of $4 \mathrm{wt} \%$. Only ${ }^{16} \mathrm{O},{ }^{238} \mathrm{U}$ and ${ }^{235} \mathrm{U}$ are included. The composition of used fuel was calculated by depleting the fresh fuel to $30 \mathrm{MWd} / \mathrm{kgHM}$ with constant power of $40 \mathrm{~kW} / \mathrm{kgHM}$ using MATREX. The tests themselves used cross section data corresponding to the beginning of step burnup and a constant flux of $4.00 \times 10^{13} \mathrm{~cm}^{-2} \mathrm{~s}^{-1}$ with the fresh fuel and $4.65 \times 10^{13} \mathrm{~cm}^{-2} \mathrm{~s}^{-1}$ with the used fuel. This leads to beginning of step power of approximately

\footnotetext{
${ }^{2}$ Some of the nuclides are duplicates used to track light elements separately based on their origin. This has no effect on solving the system.
} 
$40 \mathrm{~kW} / \mathrm{kgHM}$ in all tests. Tests with used fuel as the initial composition or as an external feed were also repeated with zero flux (i.e., with only natural decay).

The depletion tests consisted of single steps of various lengths between 1 and 1000 days. Decay-only tests were extended up to $10^{7}$ years. To keep the approximations in MATREX valid, it should never perform only a single step, but instead have at least 4-10 steps or substeps depending on the case (Gauld, 2011). To account for this, all the MATREX depletion calculations were divided to eight equidistant substeps. In the MATREX decay calculations,

the lengths of the $i^{\text {th }}$ substep was $3^{i-1} \Delta t / \sum_{j=0}^{N-1} 3^{j}$, where $\Delta t$ is the length of the step and $N$ the number of substeps. This means that each subsequent substep is three times longer than the previous one. For step lengths below $9 \times 10^{4}$ days, the number of substeps was set to eight. For step length longer than this, the number of substeps was set to the smallest value for which the length of the first substep is below 30 days. CRAM always performed a single solution for the entire length of the step. All tests used order 16 CRAM.

Test cases with time-dependent source terms are presented together with their results in Section 4.3. These calculations used cross section data for fresh fuel. As MATREX cannot handle a time-dependent source term, it is not included in the tests.

\section{Results}

In the figures that follow, the x-axis labeled as 'Atomic fraction' is the larger of the atomic fractions from the reference calculations and either 
CRAM or MATREX, whichever is being plotted. This is done to prevent large errors being pushed off the figure when the reference value is small.

All nuclides with atomic fractions below $10^{-30}$ in the reference results and in the results produced by the method being observed are ignored in the analysis and discussion. This limit corresponds to less than one atom per cubic meter. In practical applications, it should be safe to ignore all nuclides with atomic fractions below $10^{-20}$ or $10^{-15}$. Some nuclides with low atomic fractions had relative errors larger than $100 \%$ in the CRAM results. These have been plotted as $100 \%$ in the figures and marked by circling them.

\subsection{Depletion}

Fig. 1 shows the results after a single depletion step of various lengths with fresh fuel as the initial concentrations or as an external feed. MATREX used eight substeps. When both initial concentrations and external feed are present, results are a linear combination of these. This is also true in the general case as the equations are linear in respect to the initial compositions and source term. The one case where CRAM has a relative error larger than $100 \%$ is ${ }^{252} \mathrm{Cf}$ with a relative error of $150 \%$ but atomic fraction of only $4 \times 10^{-29}$. Far larger relative errors are encountered at atomic fractions below $10^{-30}$.

When the initial composition or external feed is used fuel, rather than fresh fuel, both solvers produce far more accurate results. When depleting used fuel, whether as initial composition or as an external feed, both solvers produce far more accurate results than for fresh fuel. An example of the results is shown in Fig. 2. The accuracies of both methods are only weakly affected by the step length, and even after a 1000-day step, all relative errors 
Table 2: Relative errors in decay heats calculated for the final compositions in the depletion

tests.
\begin{tabular}{|r|cc|cc|cc|cc|}
\hline $\begin{array}{r}\text { Step } \\
\text { length }\end{array}$ & \multicolumn{2}{|c|}{ Fresh fuel as initial } & \multicolumn{2}{|c|}{ Fresh fuel as feed } & \multicolumn{2}{c|}{ Used fuel as initial } & \multicolumn{2}{c|}{ Used fuel as feed } \\
& MATREX & CRAM & MATREX & CRAM & MATREX & CRAM & MATREX & CRAM \\
\hline $1 \mathrm{~d}$ & $-5.1 \times 10^{-4}$ & $-1.8 \times 10^{-15}$ & $7.4 \times 10^{-3}$ & $-3.2 \times 10^{-14}$ & $-4.9 \times 10^{-4}$ & $1.1 \times 10^{-15}$ & $6.9 \times 10^{-3}$ & $-1.9 \times 10^{-14}$ \\
$10 \mathrm{~d}$ & $2.4 \times 10^{-5}$ & $-1.2 \times 10^{-15}$ & $3.3 \times 10^{-3}$ & $-2.8 \times 10^{-14}$ & $6.4 \times 10^{-7}$ & $3.5 \times 10^{-14}$ & $3.0 \times 10^{-3}$ & $4.0 \times 10^{-15}$ \\
$100 \mathrm{~d}$ & $-3.1 \times 10^{-4}$ & $-4.4 \times 10^{-15}$ & $3.4 \times 10^{-3}$ & $-1.4 \times 10^{-14}$ & $-5.8 \times 10^{-4}$ & $1.5 \times 10^{-13}$ & $4.6 \times 10^{-3}$ & $9.6 \times 10^{-14}$ \\
$1000 \mathrm{~d}$ & $-1.5 \times 10^{-3}$ & $1.7 \times 10^{-13}$ & $1.1 \times 10^{-3}$ & $1.3 \times 10^{-13}$ & $-1.6 \times 10^{-3}$ & $2.7 \times 10^{-13}$ & $1.5 \times 10^{-3}$ & $2.0 \times 10^{-13}$ \\
\hline
\end{tabular}

from CRAM are below $10^{-6}$.

Table 2 lists the relative errors in the decay heats calculated for the final compositions in the depletion tests. While MATREX has larger errors for some of the individual nuclides in the figures, errors in the decay heats calculated for the results are still, at least for the results without external feed, still accurate enough that the errors would be insignificant in practical applications. Even for the less accurately solved external feed, errors are below $1 \%$. 

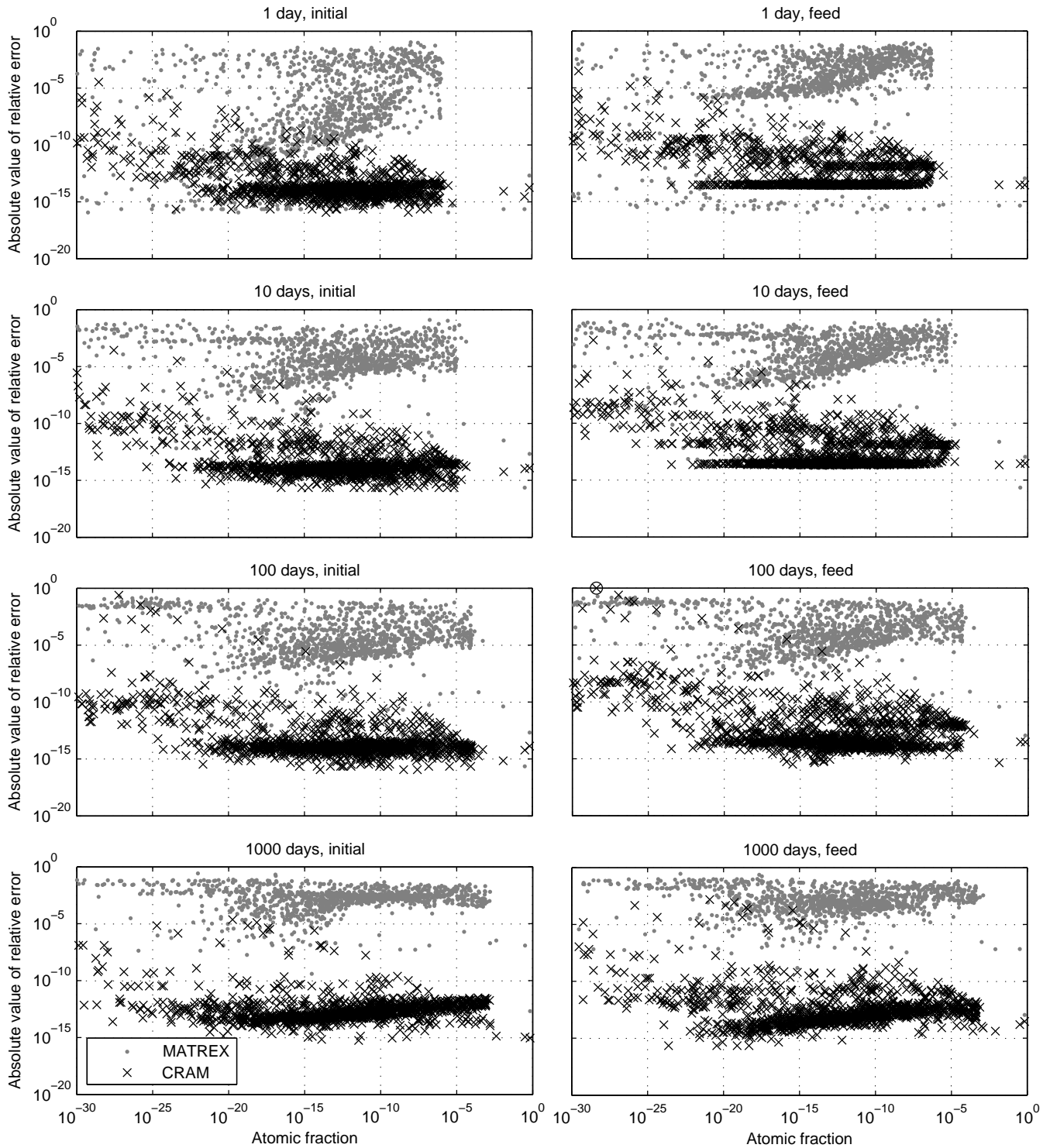

Figure 1: Relative errors for individual nuclides after a single depletion step of various lengths. MATREX used eight substeps. In the left panels, the initial composition is fresh fuel and there is no external feed. In the right panels, the initial concentrations are zero, but fresh fuel is fed in at a constant rate. Circles indicate errors that have been reduced to 1 for plotting. 

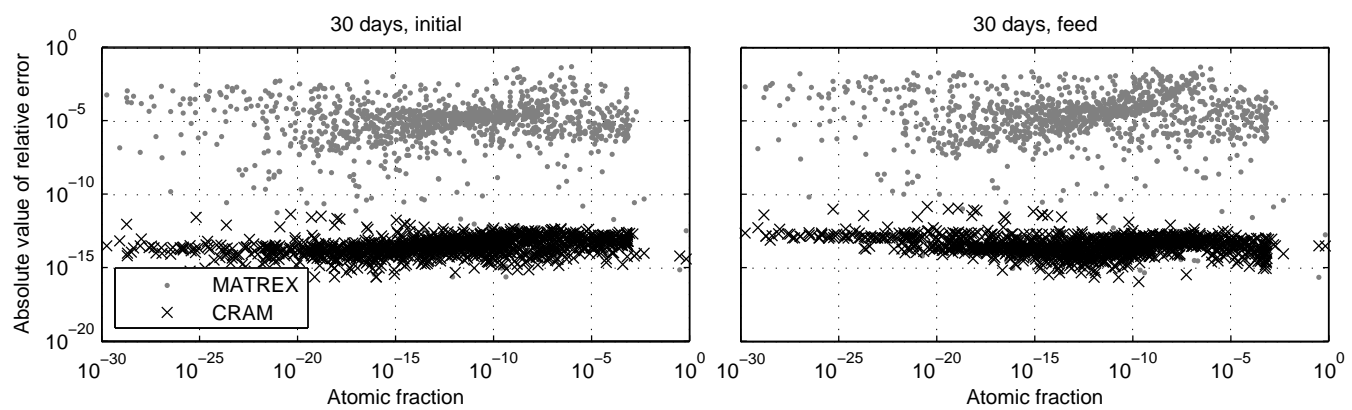

Figure 2: Relative errors for individual nuclides after a single depletion step. MATREX used eight substeps. In the left panel, the initial composition is used fuel and there is no external feed. In the right panel, the initial concentrations are zero, but used fuel is fed in at a constant rate.

\subsection{Decay}

Fig. 3 shows an example of the relative errors when used fuel is allowed to decay through a single step with substeps in MATREX. The absolute errors from CRAM remain small, and in particular, smaller than those from MATREX. This is also reflected in the decay heats calculated for the final compositions (Table 3). However, without the post-processing many nuclides have essentially unbounded relative errors. Note that the correct atomic fractions for most of these nuclides are well below $10^{-30}$, but as stated earlier, the plots use the atomic fraction from CRAM on the x-axis when it is larger than the reference value.

Enabling the post-processing for CRAM (Section 2.6) catches almost all of these errors, limiting them to $100 \%$. The only ones to make it through are a relative error of $2 \times 10^{3}$ for ${ }^{146} \mathrm{Pr}$ with a 1-day step (not shown) and a relative error of $2 \times 10^{8}$ for ${ }^{135} \mathrm{Xe}$ with a 30 -day step. The errors in the atomic fractions are $3 \times 10^{-23}$ and $3 \times 10^{-30}$, respectively, meaning that the errors are 

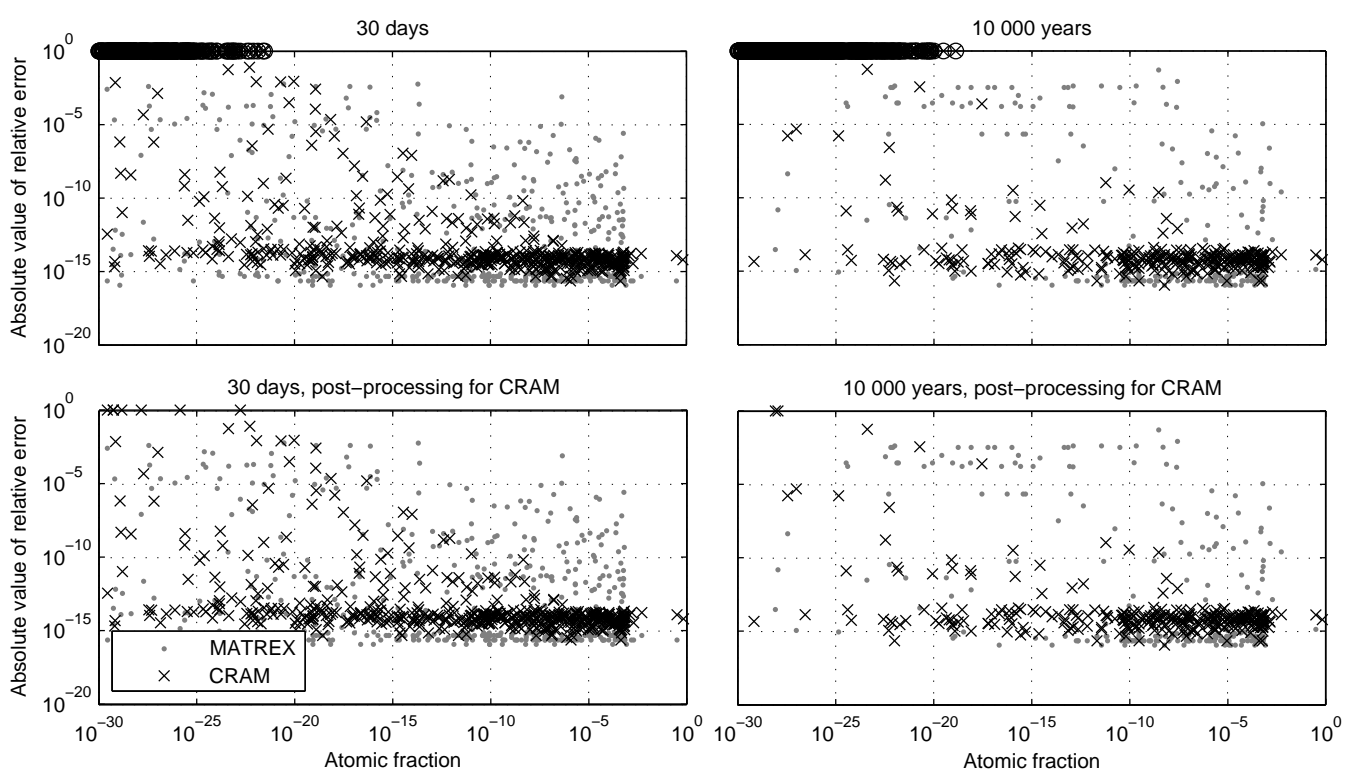

Figure 3: Relative errors with and without post-processing in CRAM for individual nuclides in decay calculations with used fuel as the initial composition. MATREX used eight substeps for the 30-day step and 12 substeps for the 10000-year step. Circles indicate errors that have been reduced to 1 for plotting.

too small to have any practical significance.

With constant feed rates and zero initial concentrations there are no nuclides whose concentration approach zero, even in decay calculations. With only decay reactions there are no long activation chains either, and CRAM produces extremely accurate results with relative errors for all nuclides below $10^{-9}$ with all step lengths (Fig. 4). MATREX, on the other hand, produced larger errors for the feed than the initial, and the relative errors for some of the nuclides get rather large with long steps. 

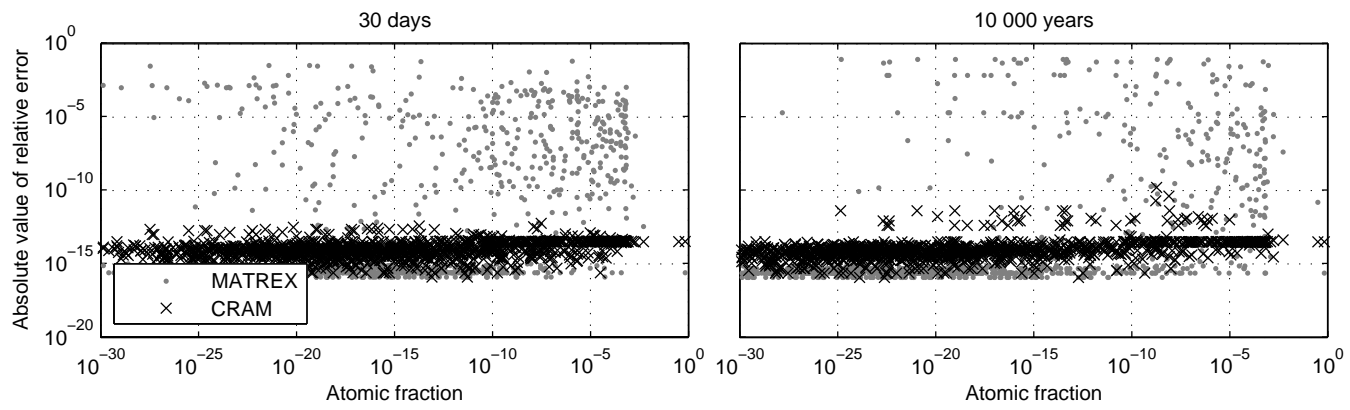

Figure 4: Relative errors for individual nuclides in decay calculations where all initial concentrations are zero, but used fuel is fed in at a constant rate. MATREX used eight substeps.

Table 3: Relative errors in decay heats calculated for the final compositions in the decay tests. Post-processing was not used.

\begin{tabular}{|r|cc|cc|}
\hline Step & \multicolumn{2}{|c|}{ Initial } & \multicolumn{2}{c|}{ Feed } \\
length & MATREX & CRAM & MATREX & CRAM \\
\hline $1 \mathrm{~d}$ & $-1.6 \times 10^{-3}$ & $-1.3 \times 10^{-14}$ & $2.3 \times 10^{-3}$ & $-9.2 \times 10^{-15}$ \\
$30 \mathrm{~d}$ & $1.0 \times 10^{-4}$ & $-1.5 \times 10^{-14}$ & $1.1 \times 10^{-2}$ & $-3.6 \times 10^{-15}$ \\
$365 \mathrm{~d}$ & $-2.2 \times 10^{-5}$ & $2.3 \times 10^{-14}$ & $1.8 \times 10^{-4}$ & $-3.0 \times 10^{-15}$ \\
$10^{4} \mathrm{a}$ & $-3.1 \times 10^{-6}$ & $3.9 \times 10^{-11}$ & $8.7 \times 10^{-5}$ & $-1.8 \times 10^{-15}$ \\
$10^{7} \mathrm{a}$ & $-6.8 \times 10^{-3}$ & $4.4 \times 10^{-9}$ & $1.5 \times 10^{-2}$ & $-1.2 \times 10^{-14}$ \\
\hline
\end{tabular}




\subsection{Time-dependent source term}

In the first test case, all initial concentrations are zero, but ${ }^{235} \mathrm{U}$ has the polynomial-in-time feed rate

$$
s(t)=c \sum_{i=1}^{m}(-1)^{i+1}\left(2 \frac{t}{\Delta t}\right)^{i},
$$

where $\Delta t=100 \mathrm{~d}$ is the step length used in the calculations, and $c$ is a constant. Tests were performed for polynomial orders (values of $m$ ) from 1 to 6. Fig. 5 illustrates the time dependence of the feed rate, and Fig. 6 shows some of the obtained results. There is a sharp cutoff for the smallest errors, but above the cutoff, the errors are very similar to those obtained for the depletion of fresh fuel (Fig. 1). Relative errors in decay heat calculated for the final compositions vary from $-4.6 \times 10^{-13}$ to $1.6 \times 10^{-8}$ for polynomial orders from one to six.

In the second test case, all initial concentrations are again zero, but each nuclide has an order $1 \ldots 6$ source term where the order $i$ coefficients are randomly selected from an uniform distribution between $-\frac{i+1}{(\Delta t)^{i}}$ and $\frac{i+1}{(\Delta t)^{2}}$. Such source terms cannot be interpreted as an external feed as they are negative as often as positive. However, they serve to demonstrate that the methodology is very general. Fig. 7 shows the relative errors in the concentrations of each nuclide. As all nuclides have a source term, the role of long activation chains is reduced, and the results are more accurate than for the above case with a

source term only for ${ }^{235} \mathrm{U}$. Relative errors in decay heat vary from $1.6 \times 10^{-14}$ to $5.9 \times 10^{-7}$ for source terms of order one to six. 


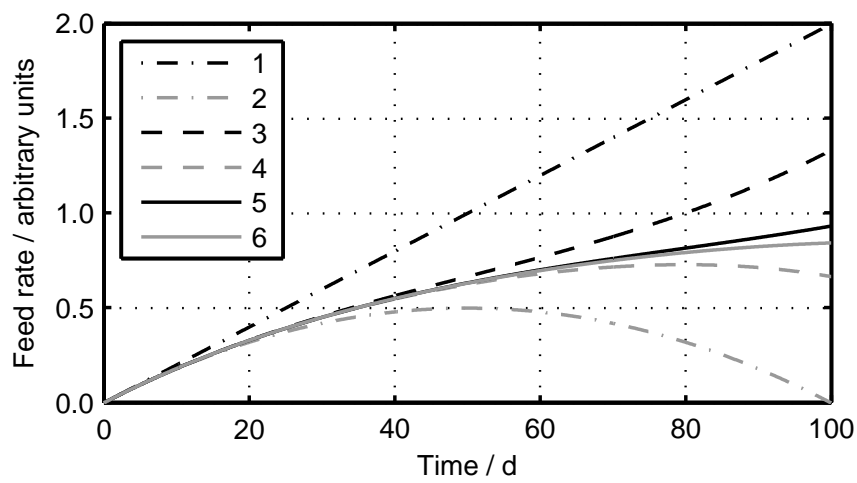

Figure 5: The time dependence of the feed rate of ${ }^{235} \mathrm{U}$ in the first test case discussed in Section 4.3
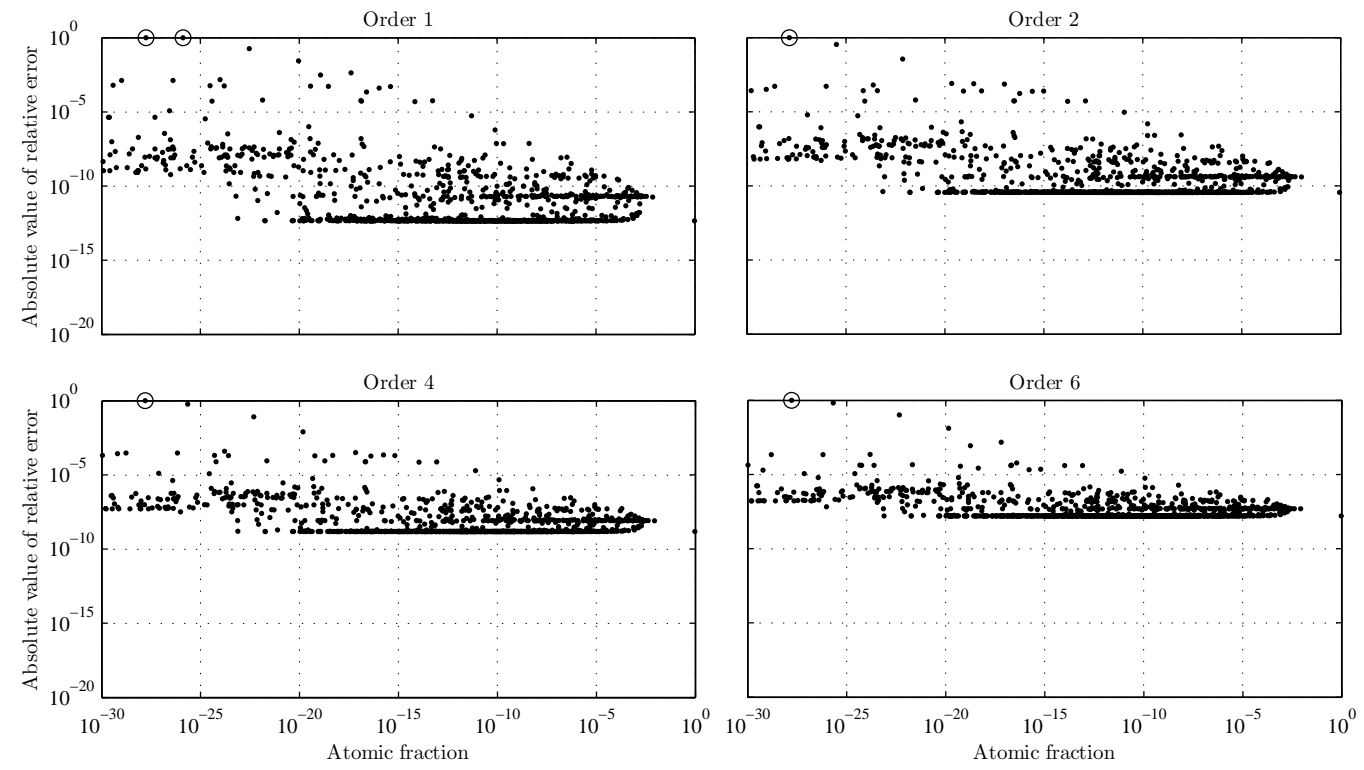

Figure 6: Relative errors after a single depletion step of 100 days when all initial concentrations are zero, but ${ }^{235} \mathrm{U}$ has the polynomial-in-time feed rate of Eq. (20). Circles indicate errors that have been reduced to 1 for plotting. 

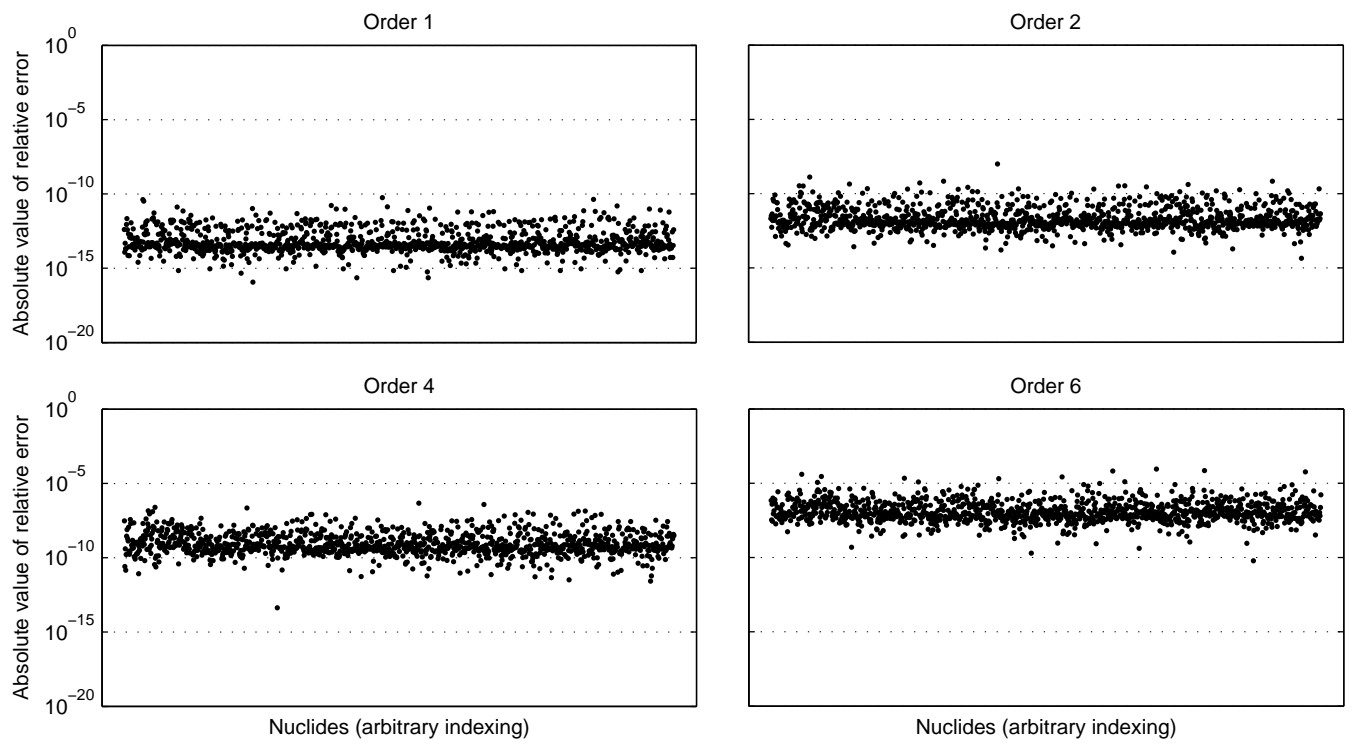

Figure 7: Relative errors after a single depletion step of 100 days when all initial concentrations are zero, but all nuclides have random polynomial source terms of different orders. 


\subsection{Running times}

Timing of the calculations was performed in serial on a single core of a AMD Opteron 6212 processor running at $2600 \mathrm{MHz}$, and the running times below have been averaged over hundreds of identical repeats. With the library data used in this work and no source term, a single depletion solution with CRAM took $41 \mathrm{~ms}$ regardless of the length of the step. MATREX took $44 \mathrm{~ms}$, $63 \mathrm{~ms}, 83 \mathrm{~ms}$ and $152 \mathrm{~ms}$ per depletion solution (i.e. substep) of 1, 10, 100, and 1000 days, respectively. In the decay calculations with zero flux, CRAM took $12 \mathrm{~ms}$ per step and MATREX $4.2-6.7 \mathrm{~ms}$ per substep of 1 day to $10^{7}$ years. The initial composition has very little effect on the running times.

Adding a constant source term for all nuclides increases the time taken by CRAM to $44 \mathrm{~ms}$ in depletion calculations and $13 \mathrm{~ms}$ decay calculations. The running time of MATREX is unaffected. Increasing the source order to $m$ with all non-zero coefficients for all nuclides adds roughly another $m$ milliseconds for CRAM. MATREX cannot use time-dependent sources. The post-processing to discard nuclides with large errors (Section 2.6) has no effect on the running time unless a source term is present. With a source term running time increases by roughly $10 \%$.

The fact that CRAM is, as far as a single solution is considered, faster for depletion but slower for decay suggests that it scales more favorably to large systems and consequently less favorably to small ones. To test this, some of the timings were repeated with a smaller library of 255 nuclides and 5035 transitions. In these tests, CRAM took $4.6 \mathrm{~ms}$ per depletion step, while MATREX without substeps only took 1.5, 2.0, 2.9, and 3.5 ms for depletion steps of 1, 10, 100, and 1000 days, respectively. This fits well with the idea 
of CRAM scaling better to large problems, but it might also be related to the small library containing relatively few short-lived nuclides.

Decreasing the order of CRAM reduces running time by about $5 \%$ per order. However, this also rapidly decreases accuracy. In most applications lower accuracy might be acceptable, but so should the running time of order 16 CRAM.

The above running times are for the solver functions in ORIGEN. ORIGEN supplies the solver with data in the format used by the MATREX solver. The running times for CRAM include $3.6 \mathrm{~ms}$ in the depletion cases and $1.8 \mathrm{~ms}$ in the decay cases for converting the data to a compressed row storage (CRS) sparse matrix form. This could be avoided in a code where reaction rates are stored in a format directly usable by the CRAM implementation.

\section{Discussion and conclusions}

Relative errors in the decay heats for the compositions calculated with CRAM were below $10^{-9}$ in all tests without time-dependent source term and below $10^{-6}$ even with an order six polynomial source term. With MATREX, the relative errors were at most $6.8 \times 10^{-3}$ without external feed, and $1.5 \times 10^{-2}$ with only external feed. This is still below the uncertainties in experimental results (Ilas et al., 2014), which suggests that either method should generally be accurate enough for integral quantities, such as decay heat, activity, radiotoxicity, total cross-sections, and all the transport parameters determined by the total cross sections. However, only a few cases were tested, and only decay heat was observed, so the tests are by no means comprehensive enough to determine how large the worst case errors might be. 
Even integral quantities may be dominated by a single nuclide and sometimes the concentrations of individual nuclides are required directly. The largest errors for individual nuclides thus serve as the upper limit for all errors in a particular case regardless of application. Note, however, that the solver-related errors measured and discussed in this work do not include the uncertainties propagated from nuclear data or other input.

\subsection{Depletion without external feed}

With used fuel as the initial composition, all concentrations calculated with CRAM are accurate to at least six digits for step lengths up to 1000 days, which was the longest step tested. When starting from fresh fuel instead, the great majority of the results are still correct to six or more digits, but there are some outliers, particularly at smaller atomic fractions, with larger relative errors. However, the relative errors only become notable for nuclides with concentrations so low that they are unlikely to affect anything. As noted by Isotalo and Aarnio (2011), these outliers correspond to long chains of captures and $\beta^{-}$-decays. In used fuel, such long chains have far less significance as chains starting from the intermediate nuclides dominate. This leads to better accuracy with both CRAM and MATREX.

It should be noted that the results with used fuel as the initial concentrations or external feed are more relevant in most applications than those starting with fresh fuel. This is due to fuel only being fresh before the first step of depletion. The concentrations of the intermediate nuclides do not need to be high for the contributions from them to dominate in long chains. 


\subsection{Decay without external feed}

When the concentration of an initially present nuclide approaches zero, relative errors produced by CRAM can become arbitrarily large. This makes the decay calculations the weakest point of CRAM, but the largest absolute errors are still smaller than those from MATREX. Note that similar errors are also possible with non-zero flux if the initial composition contains shortlived nuclides that are far above their secular equilibrium concentrations.

In the tests of this work, the post-processing to remove low concentration nuclides with large errors (Section 2.6) worked fairly well and zeroed almost all atomic densities with relative errors larger than $100 \%$. However, as the post-processing does not account for errors inherited from other nuclides, it cannot be relied to work equally well in the general case. In particular, it can never detect the errors for a nuclide whose initial atomic density is zero. Options to more reliably detect these errors may be studied in the future. However, if the concentrations of nuclides with very low atomic densities are of concern, the correct approach might be to use a higher order CRAM (Pusa, 2014), which may be implemented into ORIGEN at a later date.

\subsection{Accuracy of the results for external feed}

As predicted by theory, the results from CRAM for a constant feed are as accurate as those for the decay and depletion of initial nuclides. In the de-

cay tests, the results for external feed appear to be more accurate than those without, but this does not contradict the theory suggesting "only" equal accuracy. The results are equally accurate for all nuclides whose concentrations do not approach zero, as well as for all nuclides whose concentrations do. However, with constant feed rates and zero initial concentrations, there are 
no nuclides whose concentrations would approach and thus no large errors that would occur for such nuclides.

In the tests with a random time-dependent source term, errors are clustered around the level that would be expected based on the derivatives of the rational approximation (Table 1). In the tests with a polynomial-in-time feed rate for ${ }^{235} \mathrm{U}$ only, there are larger errors for some nuclides, but they correspond to those observed in the fresh fuel tests and seem unrelated to the handling of the source term. The smallest errors are also an order of magnitude above those in the derivatives ${ }^{3}$, but there is no reason to expect an exact agreement, and the errors are within the variation observed with a random source.

Since only the smallest errors are significantly increased, a low enough order source term does not significantly affect accuracy in practical applications. With order $k$ CRAM, the current implementation in ORIGEN accepts source terms up to order $k / 2-2$ or zero, whichever is higher. Thus the highest is an order 6 source term with order 16 CRAM.

If an exponential source term (Eqs. (10) and (11)) was implemented, it should not be affected by the same loss of accuracy as the polynomial one. However, if some of the exponential components decrease too much during a step, they would lead to similar errors as in the decay tests.

\footnotetext{
${ }^{3}$ This is also true with the order 4 and 6 source terms, even if this is not apparent from Fig. 6 where the higher order components in the source term are relatively small.
} 


\subsection{Running times}

In the depletion tests with typical step lengths of 10-100 days, CRAM required 25-50\% less CPU time for a single solution than MATREX did, depending on step length and source term. In decay tests, on the other hand, CRAM took 100-200\% longer than MATREX. Without the additional data processing required in CRAM to convert the input data to a CRS format, depletion solutions with CRAM would take 35-60\% less time and decay solutions 60-160\% more time than those with MATREX.

However, all the MATREX results presented in Section 4 were calculated with eight substeps, requiring eight solutions per step. This means that CRAM was significantly faster in all tests. Without substeps, the accuracy of MATREX would be lower, especially in the decay tests and for the external feeds. Using additional substeps could also improve the accuracy to some degree. Furthermore, if the subsequent steps of a multistep calculation are similar enough in terms of length and reaction rates, substeps may not be needed to reach full accuracy. Because there is no clear answer to how many substeps are needed or should be used with MATREX, there is no general rule for the ratio of the running times of the methods in practical applications. CRAM should, however, always be faster unless the substeps in MATREX can be omitted.

Isotalo and Aarnio (2011) reported a significantly different ratio of running times of CRAM and the solver of ORIGEN2, making the solver of ORIGEN2 much faster on a per-solution basis. While there are some differences between the solver of ORIGEN2 and the up-to-date MATREX, they should not have any significant effect on the running times. Instead, the dif- 
ference can be explained by two main factors. First, their tests used library data with fewer nuclides and reactions. Second, the CRAM implementation in this work is faster. When the same code used by Isotalo and Aarnio (2011) was used for the test cases of this work, it was slower by a factor of eight.

This serves as a reminder that CRAM and MATREX are complex enough that their running times are highly dependent on the efficiency of the implementation. How optimal either of the solvers in this work is was not studied.

\subsection{Conclusions}

A method for including a source term in decay and depletion calculations with the Chebyshev rational approximation method (CRAM) was developed and implemented into ORIGEN in the SCALE suite. The methodology allows accurate modeling of external feed of nuclides with polynomial or exponentially decaying time dependence and may also be applicable with other matrix exponential methods for depletion calculations. In addition to being able to handle time-dependent feeds and adjoint calculations, the new CRAM solver is more accurate, and in typical depletion calculations with the SCALE 6.1 libraries also faster, than the original solver. In decay cases with fewer transitions or when using libraries that include fewer nuclides and reactions, the original solver may under some conditions be faster, but CRAM should still be preferable in most, if not all, applications. The new CRAM solver will be available with the release of SCALE 6.2.

\section{Acknowledgements}

Funding from SAFIR2014, the Finnish Research Program on Nuclear Power Plant Safety, and the Nuclear Energy Advanced Modeling and Simula- 
tion (NEAMS) program of the U.S. Department of Energy is acknowledged.

\section{References}

Cetnar, J., 2006. General Solution of Bateman Equations for Nuclear Transmutations. Ann. Nucl. Energy 33, 640-645. doi:10.1016/j.anucene.2006.02.004.

Fousse, L., Hanrot, G., Lefèvre, V., Pélissier, P., Zimmermann, P., 2007. MPFR: A Multiple-Precision Binary Floating-Point Library with Correct Rounding. ACM Trans. Math. Software 33, 13:1-13:15. doi:10.1145/1236463.1236468

Gauld, I.C., Radulescu, G., Ilas, G., Murphy, B.D., Williams, M.L., Wiarda, D., 2011. Isotopic Depletion and Decay Methods and Analysis Capabilities in SCALE. Nuclear Technology 174, 169-195.

Gauld I.C. 2011. ORIGEN: Depletion Module to Calculate Neutron Activation, Actinide transmutation, Fission Product Generation, and Radiation Source Terms. In SCALE: A Comprehensive Modeling and Simulation Suite for Nuclear Safety Analysis and Design, ORNL/TM-2005/39, Version 6.1. Available from Radiation Safety Information Computational Center at Oak Ridge National Laboratory as CCC-785.

Hairer, E., Wanner, G., 1991. Solving Ordinary Differential Equations II. Stiff and Differential-Algebraic Problems. Springer Series in Computational Mathematics 14, Springer-Verlag 1991. Second Edition. 
Ilas, G., Gauld, I.C., Liljenfeldt, H., 2014. Validation of ORIGEN for LWR used fuel decay heat analysis with SCALE. Nucl. Eng. Des. 273, 58-67. doi:10.1016/j.nucengdes.2014.02.026

Isotalo, A.E., Aarnio, P.A., 2011. Comparison of Depletion Algorithms for Large Systems of Nuclides. Ann. Nucl. Energy. 38, 261-268. doi:10.1016/j.anucene.2010.10.019

Isotalo, A.E., 2013. Computational Methods for Burnup Calculations with Monte Carlo Neutronics. Doctoral Dissertation, Aalto University. ISBN: 978-952-60-5398-1.

Li, X.S., 2005. An Overview of SuperLU: Algorithms, Implementation, and User Interface. ACM Trans. Math. Softw. 31, 302-325. doi:10.1145/1089014.1089017

Oak Ridge National Laboratory, 2011. SCALE: A Comprehensive Modeling and Simulation Suite for Nuclear Safety Analysis and Design, ORNL/TM2005/39, Version 6.1. Available from Radiation Safety Information Computational Center at Oak Ridge National Laboratory as CCC-785.

Pusa, M., Leppänen, J., 2010. Computing the Matrix Exponential in Burnup Calculations. Nucl. Sci. Eng. 164, 140-150. doi:10.13182/NSE09-14

Pusa, M., 2013a. Accuracy Considerations For Chebyshev Rational Approximation Method (CRAM) in Burnup Calculations. M\&C 2013, Sun Valley, Idaho, May 5-9, 2013.

Pusa, M., 2013b. Numerical Methods for Nuclear Fuel Burnup Calculations. Doctoral Dissertation, Aalto University. ISBN: 978-951-38-8000-2 
Pusa, M., 2014. Higher-Order Chebyshev Rational Approximation Method (CRAM). PHYSOR 2014. Kyoto, Japan, September 28 - October 3, 2014.

Higham, N.J., 2008. Functions of Matrices, Theory and Computation. Society for Industrial and Applied Mathematics. ISBN: 978-0-898716-46-7

Williams, M.L., 1978. Development of Depletion Perturbation Theory for Coupled Neutron/Nuclide Fields. Nucl. Sci. Eng. 70, 20-36. 10.13182/NSE79-3 